Classification

Physics Abstracts

$52.75 \mathrm{Hn}-52.80 \mathrm{Pi}-52.70 \mathrm{Kz}$

\title{
Torche à plasma à excitation micro-onde : deux configurations complémentaires
}

\author{
A. Ricard $\left({ }^{1}\right)$, L. St-Onge $\left({ }^{2}\right)$, H. Malvos $\left({ }^{3}\right)$, A. Gicquel $\left({ }^{4}\right)$, J. Hubert $\left({ }^{2}\right)$ et M. Moisan $\left({ }^{2}\right)$ \\ $\left({ }^{1}\right)$ Laboratoire de physique des gaz et des plasmas, Université Paris-Sud, 91405 Orsay, France \\ $\left({ }^{2}\right)$ Groupe de physique des plasmas, Université de Montréal, C. P. 6128, Succursale Centre-ville, \\ Montréal H3C 3J7, Québec \\ $\left({ }^{3}\right)$ Centre d'essais et de recherches sur les utilisations du gaz, Gaz de France, 93211 La Plaine \\ Saint-Denis Cedex, France \\ $\left({ }^{4}\right)$ Laboratoire d'ingénierie des matériaux et des hautes pressions, CNRS-UPR 1311, Avenue J. \\ B. Clément, 93430 Villetaneuse, France
}

(Reçu le 27 février 1995, révisé le 17 mai 1995, accepté le 19 mai 1995)

Résumé. - Cet article décrit la "flamme" obtenue à la pression atmosphérique par une décharge micro-onde $(2,45 \mathrm{GHz})$ à partir de deux configurations de torche à plasma utilisant le même dispositif d'adaptation d'impédance. Ces deux torches se différencient extérieurement par la forme du conducteur portant le champ micro-onde relativement à l'injection du gaz plasmagène : une buse conique avec une injection axiale des gaz (système TIA) dans un cas et une tige avec une injection de gaz qui lui est coaxiale (système torche-tige) dans l'autre cas. La TIA donne lieu à un dard bien défini de forte luminosité, avec un maximum de densité de particules chargées au sortir de la buse; la torche-tige conduit à un plasma de moins grande densité mais de plus grande température au voisinage immédiat de l'applicateur. Le plasma de la torche-tige est plus près de l'équilibre thermodynamique local (ETL) que celui de la TIA.

\begin{abstract}
This paper describes the "flame" obtained at atmospheric pressure by a microwave discharge $(2.45 \mathrm{GHz})$ produced using two different plasmia torch configurations sharing the same impedance matching device. These two torches differ externally by the shape of their microwave field applicator relatively to the way the carrier gas is injected: a conical nozzle with axial gas injection (the "TIA" system) in one case. and a rod with coaxial gas injection (the "rod-torch" system) in the other. The TIA produces a well defined tongue with a high luminosity and a maximum of charged particle density at the nozzle's exit, while the rod-torch leads to a lower density plasma but to a higher gas temperature in the vicinity of the applicator. The plasma of the rod-torch is closer to local thermodynamic equilibrium (LTE) than that of the TIA.
\end{abstract}




\section{Introduction}

Une torche à plasma est de façon générale un dispositif où la décharge est produite à partir d'un gaz (ou mélange de gaz) en écoulement rapide, à la pression atmosphérique ou à pression réduite. Dans les deux torches que nous allons décrire, le plasma se forme à l'extrémité d'un conducteur qui porte le champ électrique micro-onde. L'avantage de ce type d'excitation par rapport aux systèmes à arcs tient à l'absence de contre-électrode ou de plan conducteur de référence et à la formation d'une "flamme" $\left({ }^{1}\right)$ plus stable ; en comparaison de la décharge à couplage inductif qui fonctionne dans le domaine des fréquences radio (e.g., $13 \mathrm{MHz}$ ), le plasma des deux torches présentées est de diamètre beaucoup plus faible.

Les wurches à plasma peuvent être utiles notamment pour le traitement de surface (par exemple, le dépôt de matériaux par projection [1], la formation de couches minces de diamant $[2,3]$ ), pour réaliser des réactions chimiques (par exemple, la destruction de gaz toxiques [4] ou affectant la couche d'ozone [5]) et pour l'analyse chimique $[6,7]$.

Nos travaux sur les plasmas à la pression atmosphérique avaient jusqu'à maintenant pour objet unique les décharges entretenues par des ondes de surface électromagnétiques $[8,9]$. Ces décharges ont la caractéristique d'être produites dans un tube diélectrique. Lorsque celui-ci est d'un diamètre interne suffisamment petit, on obtient à cette pression, avec la plupart des gaz, un filament de plasma centré sur l'axe du tube, filament que l'on peut faire sortir partiellement à l'air libre lorsque l'excitateur est assez près de l'extrémité ouverte du tube : cette configuration de décharge constitue une torche à plasma. L'inconvénient de ce dispositif est que sa flamme augmente fort peu en densité de charges et en température (donc en enthalpie) lorsqu'on accroît la puissance micro-onde : la plus grande partie de cette puissance est plutôt dépensée dans le plasma contenu à l'intérieur du tube à décharge pour en augmenter la densité, le diamètre et, le cas échéant, la longueur. L'augmentation de la densité de puissance absorbée entraîne en même temps une détérioration accrue du tube à décharge, à partir de températures plus ou moins élevées suivant la nature de son matériau (silice fondue et céramique le plus souvent). Pour obtenir des densités de plasma plus grandes et des températures de gaz plus élevées en vue d'opération comme le dépôt de couches minces de diamant, il nous fallait donc trouver un autre type de torche, de préférence alimentée par des micro-ondes afin de bénéficier de nos connaissances et équipements dans le domaine. L'article qui suit décrit les résultats de ces travaux exploratoires.

L'originalité du dispositif que nous présentons tient aux éléments suivants : i) l'alimentation en puissance micro-onde de la torche se fait par un guide d'onde (par opposition à une ligne coaxiale), ce qui permet, le cas échéant, d'aller à des puissances dépassant largement le kilowatt à $2,45 \mathrm{GHz}$; ii) le même système d'adaptation d'impédance peut accueillir deux configurations différentes d'applicateur micro-onde, l'une en forme de buse conique avec injection axiale du gaz plasmagène, dite TIA (pour torche à injection axiale) [10], et l'autre en forme de tige avec une injection de gaz qui lui est coaxiale, cette seconde configuration étant ici présentée pour la première fois. Les plasmas obtenus dans ces deux cas sont fort différents quant à leur forme et à leurs propriétés.

Le système d'adaptation d'impédance commun aux deux configurations a déjà fait l'objet d'une étude approfondie dans laquelle nous décrivons un circuit électrique équivalent et où l'on montre comment accroître la stabilité de l'accord d'impédance quant aux changements dans les conditions opératoires [10]. Il restait à présenter les propriétés du plasma de ces torches, ce à

$\left({ }^{1}\right)$ La flamme désigne dans notre article la partie lumineuse de ce que produit la décharge, sans référence à une combustion. Nous verrons que cette flamme se décompose en un dard et en un panache. 


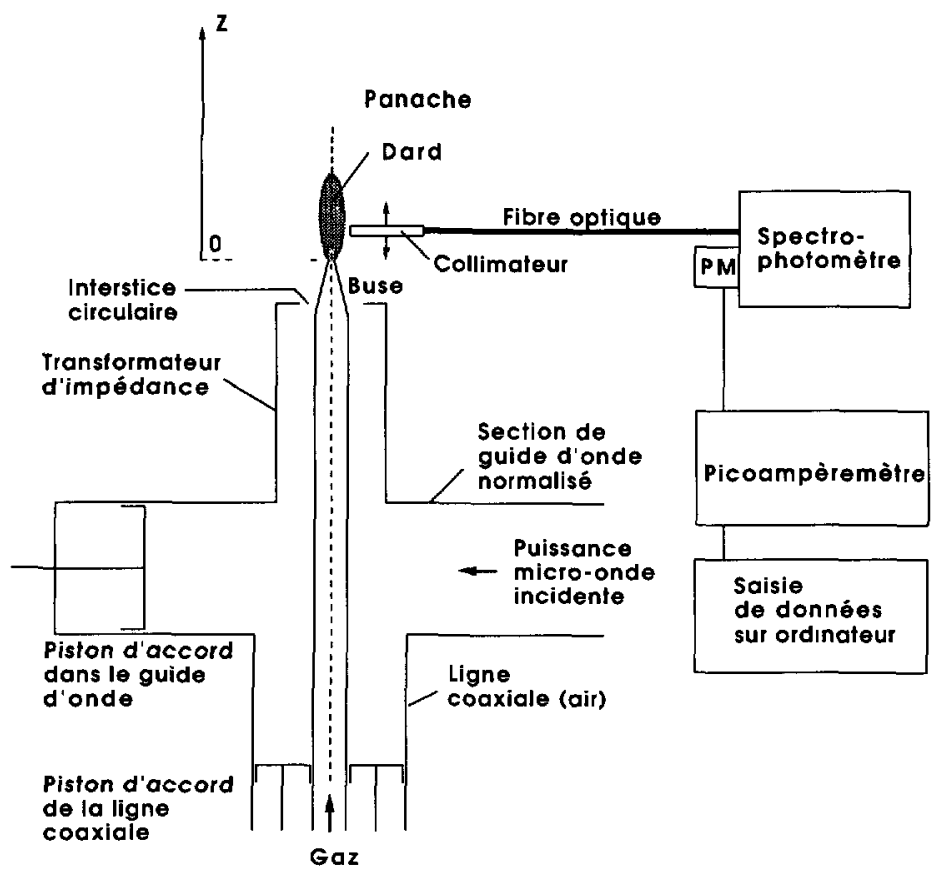

Fig. 1. - Représentation schématique du dispositif d'excitation de la torche à plasma, de sa flamme et du montage pour le diagnostic de la densité électronique et de la température du gaz par spectroscopie d'émission. L'applicateur de champ micro-onde est représenté avec la buse de la configuration TIA. Le système d'adaptation d'impédance comprend le transformateur d'impédance et deux pistons d'accord, l'un dans la ligne coaxiale se terminant à l'interstice circulaire et lautre situé dans la partie du guide d'onde prolongeant l'amenée de puissance du générateur. La forme de la flamme, représentée de façon schématique, montre l'existence de deux régions lumineuses, le dard et le panache ; noter le repère de position axiale $z$. Pour permettre une étude en fonction de $z$ de l'émission lumineuse, on a disposé un collimateur de $1 \mathrm{~mm}$ de diamètre transversalement à l'axe de la flamme.

[Schematic drawing of the plasma torch excitation device, of its flame, and of the arrangement used for electron density and gas temperature measurements through emission spectroscopy. The microwave field applicator is the conical nozzle of the TIA configuration. The impedance matching system includes an impedance transformer and two tuning stubs, one along the coaxial line terminated by the applicator and the other located in the extension of the waveguide feed. The shape of the flame, approximately drawn, shows the existence of two luminous regions, the tongue and the plume; note the reference point for the axial position $z$. To provide spatial resolution of the light emission along $z$, we have placed a collimator $1 \mathrm{~mm}$ in diameter transversely to the flame axis.]

quoi s'emploiera le présent article. Le texte qui suit se divise en trois parties : la description du montage expérimental (paragraphe 2), la présentation des résultats expérimentaux (paragraphe 3 ), et une discussion assortie d'une conclusion (paragraphe 4).

\section{Montage expérimental}

2.1. Description du dispositif D'excitation Du Plasma. - La figure 1 donne un schéma d'ensemble de la source de plasma qui comprend l'applicateur de champ (la partie qui émerge de l'interstice circulaire) et le système d'adaptation d'impédance, situé entre l'applicateur et la ligne de transmission amenant la puissance micro-onde du générateur. Ce système d'adaptation 
d'impédance [10] a pour première mission de convertir le champ du mode fondamental $\mathrm{TE}_{10} \mathrm{du}$ guide rectangulaire en celui du mode fondamental TEM de la ligne coaxiale acheminant le flux de puissance jusqu'à l'applicateur ; sa deuxième tâche est de rendre l'adaptation d'impédance avec le générateur le plus insensible possible aux variations des conditions de décharge (nature et débit du ou des gaz, y compris l'injection de produits à traiter) et de la puissance micro-onde. Ceci s'accomplit grâce à l'incorporation d'un transformateur d'impédance [10] entre le guide d'onde et l'interstice circulaire (Fig. 1) : un bon transformateur fait en sorte que la puissance réfléchie vers le générateur (et donc la puissance absorbée dans le plasma) soit peu affectée lorsqu'on modifie les conditions de décharge de façon importante, ce qui concrètement renforce la capacité du plasma à résister à l'extinction quand on introduit successivement diverses substances dans le gaz plasmagène.

La figure 1 montre aussi de façon schématique la flamme de la torche; celle-ci doit être dirigée verticalement pour être stable par suite des effets naturels de convection. On remarquera l'existence de deux régions lumineuses, le dard, de plus grande intensité, et le panache, plus diffus et situé plus loin de l'applicateur.

La figure 2 représente les deux applicateurs, obligatoirement conducteurs, que nous avons utilisés et donne leurs dimensions typiques. La buse de la TIA, de forme conique (Fig. 2a), est généralement faite de cuivre ou de laiton alors que la tige de la torche-tige (Fig. 2b) est en tungstène ; dans nos conditions habituelles de fonctionnement, l'extrémité de la TIA s'échauffe peu ou pas au contraire de celle de la torche-tige, d'où le choix des matériaux : la meilleure conductivité avec un matériau peu coûteux et facilement usinable dans le premier cas, et le meilleur conducteur réfractaire dans le second cas. Noter la manière dont se fait l'arrivée du gaz plasmagène : de façon centrale pour la TIA mais au moyen d'une "douche" coaxiale à la tige (Fig. 2c) pour la torche-tige. On passe aisément d'une configuration à l'autre, la buse et la base de la tige étant rattachées au conducteur central de l'applicateur par un simple filetage.

Deux remarques de nature expérimentale s'imposent. La première est que l'extrémité de la buse de la TIA, pour un débit de gaz supérieur à environ $1 \mathrm{l} / \mathrm{min}$ (aux conditions standards) s'échauffe si peu que l'on peut généralement la toucher avec ses doigts dès que l'on a coupé I'alimentation micro-onde (prudence quand même!) ; en revanche, l'extrémité de la torche-tige est portée à haute température, au point dans certains cas d'entrer en fusion. La seconde remarque concerne l'origine de la configuration de la torche-tige : nous avons voulu savoir si la décharge à l'extrémité de la buse de la TIA résultait d'un simple effet de concentration d'énergie micro-onde, que l'applicateur soit conducteur ou non. Il s'avère que l'applicateur doit être conducteur, à la différence des torches à plasma à onde de surface.

2.2. DESCRIPTION D'ENSEMBLE DU MONTAGE. - La torche est alimentée par un générateur micro-onde fonctionnant à $2,45 \mathrm{GHz}$ et pouvant donner jusqu'à $3 \mathrm{~kW}$ (taux d'ondulation résiduelle inférieur à $3 \%$ ). Nous avons considéré que la puissance absorbée par le plasma s'obtenait en soustrayant de la puissance incidente la puissance réfléchie à l'entrée du dispositif (Fig. 1), ce qui revient à négliger les pertes de rayonnement micro-onde et les pertes dans le circuit d'accord d'impédance [10]. L'applicateur et le plasma doivent néanmoins être contenus dans une cage de Faraday pour protéger l'opérateur du rayonnement micro-onde. rayonnement qui décroît cependant rapidement avec la distance à l'applicateur.

Le débit de gaz était en général de $5 \mathrm{l} / \mathrm{min}$ aux conditions standards; un débit inférieur au litre par minute entraînait une détérioration de l'applicateur de champ, surtout dans le cas de la tige. Nous avons travaillé avec de l'argon et de l'hélium auxquels, dans certains cas, nous avons ajouté de l'hydrogène, jusqu'à $20 \%$ en volume du débit total. Au-delà de ces pourcentages d'hydrogène, la décharge était moins stable. 


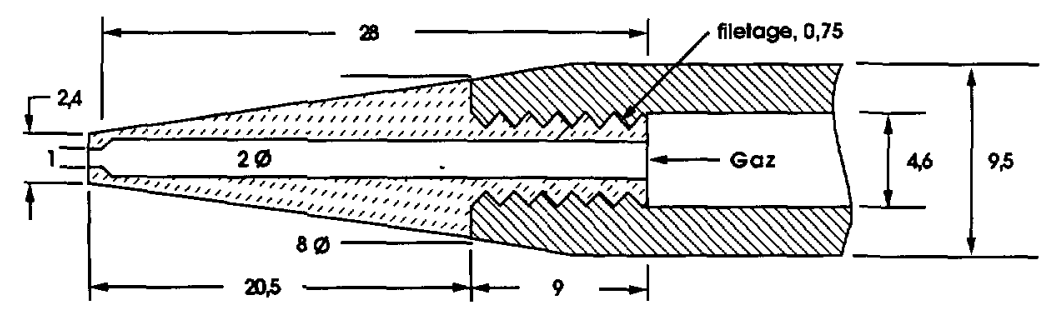

a)

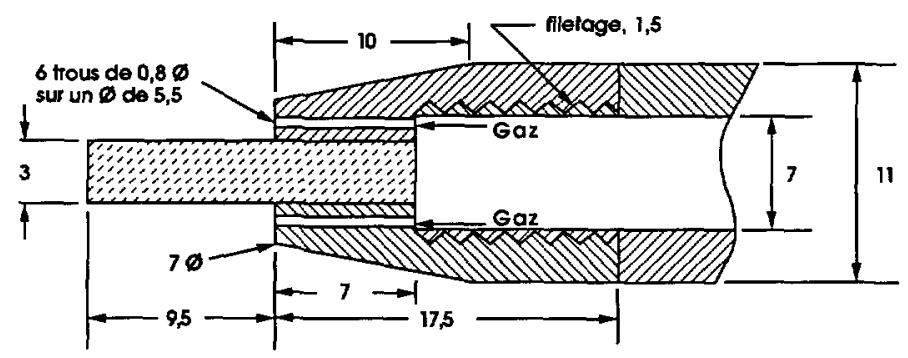

b)
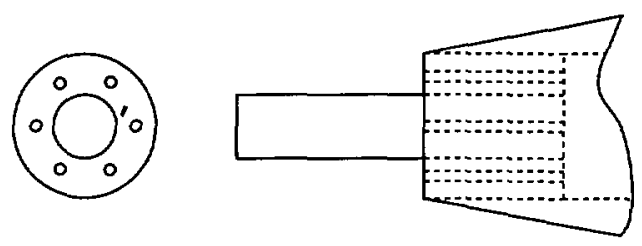

c)

Fig. 2. - Dessin en coupe des deux applicateurs de champ micro-onde avec leurs dimensions (en $\mathrm{mm}$ ) ; a) la buse (cuivre) de la torche à injectıon axıle (TIA) ; b) la tige pleine (tungstène) avec c) son système d'injection coaxiale de gaz (douche) constituant la torche-tıge.

[Cross-sectional drawing of both microwave field applicators with their dimensions; a) the nozzle (copper) of the axial injection torch (TIA); b) the solid rod (tungsten) and c) its coaxial gas injection system (shower) constituting the rod-torch.]

La figure 1 montre de façon schématique le système de diagnostic optique utilisé pour déterminer la température du gaz et la densité du plasma à différentes positions le long de la flamme.

\subsection{Description des MÉthodes de diagnostic}

2.3.1. Température du gaz. - L'analyse spectroscopique de la flamme des deux torches a révélé la présence. sans doute du fait de l'air ambiant, de bandes de $\mathrm{N}_{2}, \mathrm{NO}, \mathrm{OH}$ mais aussi de $\mathrm{CN}$, dans ce dernier cas probablement en raison d'impuretés d'hydrocarbure dans les bouteilles de gaz. Nous avons alors décidé de mettre à profit ces émissions pour déterminer la température du gaz de la flamme en supposant que celle-ci était en équilibre avec la température $T_{\mathrm{R}}$ des systèmes rotationnels de ces bandes : comme nous le verrons, le plasma des deux torches n'est pas en ETL de sorte qu'il n'y a pas une température unique pour caractériser le plasma.

L'émission lumineuse a été recueillie à l'aide d'un collimateur de $1 \mathrm{~mm}$ de diamètre dont nous avons ajusté la position transversalement à l'axe de la décharge pour obtenir un maximum de luminosité. Il convient de noter que la turbulence de la flamme, y compris dans le panache, était si faible que les différentes émissions enregistrées n'en ressentaient pas l'effet ; la stabilité de la 


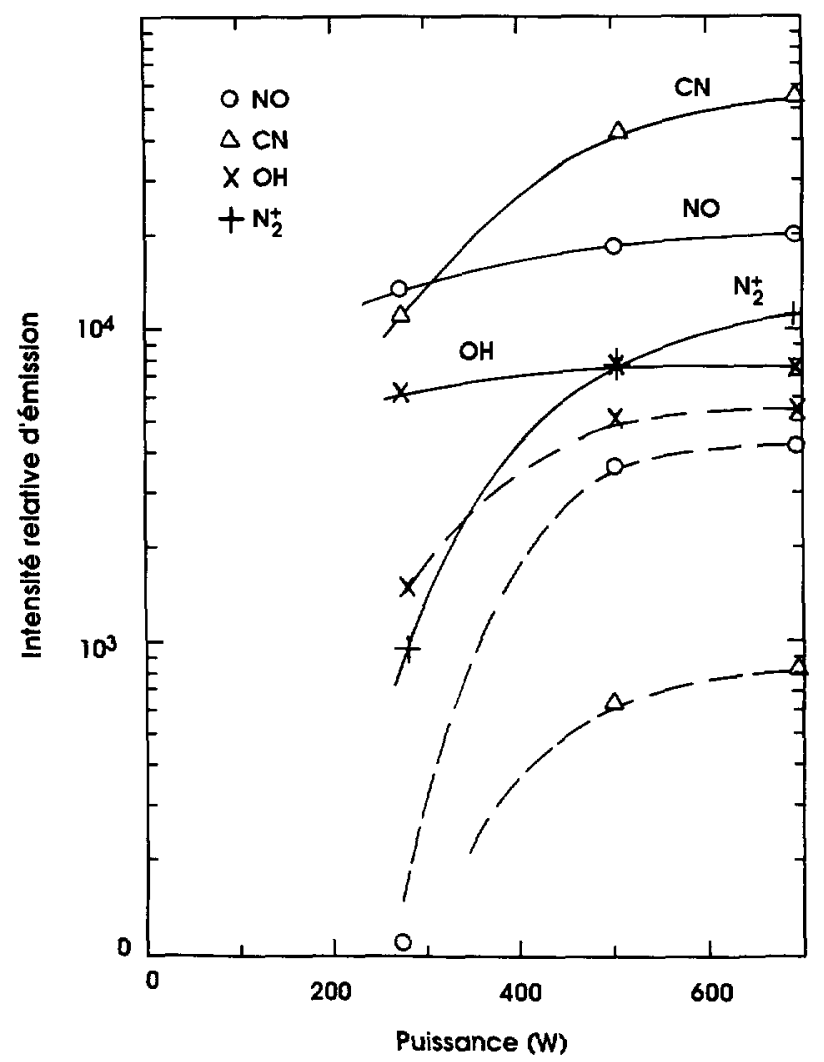

Fig. 3. - Intensité relative d'émission de la tête de bande de quatre structures rotationnelles caractéristiques de la flamme de la torche-tige en fonction de la puissance micro-onde absorbée, dans le dard en $z=2 \mathrm{~mm}$ (trait plein) et dans le panache en $z=20 \mathrm{~mm}$ (tirets), en injectant de l'argon par la douche à $4,8 \mathrm{l} / \mathrm{min}$.

[Relative emission intensity of the band head of four rotational structures characterizing the flame of the rod-torch as a function of the absorbed microwave power, in the tongue at $z=2 \mathrm{~mm}$ (full line) and in the plume at $z=20 \mathrm{~mm}$ (dashed line) upon injecting argon through the shower at a rate of $4.8 \mathrm{l} / \mathrm{min}$.]

décharge permettait des enregistrements pouvant durer jusqu'à dix minutes. Pour déterminer laquelle des émissions utiliser, nous avons d'abord enregistré la variation des intensités des têtes de bandes de $\mathrm{CN}, \mathrm{NO}, \mathrm{N}_{2}^{+}$et $\mathrm{OH}$ en fonction de la puissance micro-onde. La figure 3 montre, à titre d'exemple, ces émissions pour la torche-tige avec de l'argon injecté par la douche. La mesure a été effectuée dans le dard à une distance axiale $z=2 \mathrm{~mm}$ à partir de l'applicateur et dans le panache à $z=20 \mathrm{~mm}$. Les bandes de $\mathrm{N}_{2}^{+}$sont intenses dans le dard mais n'apparaissent pour ainsi dire pas dans le panache. Par contre, l'intensité de $\mathrm{OH}$ diminue peu du dard au panache, de seulement $30 \%$ au-delà de $500 \mathrm{~W}$. Ces deux systèmes de bandes sont, pour les deux torches, d'une intensité très supérieure à celle de l'émission du gaz plasmagène. Ce phénomène indique l'incorporation certaine d'air et d'humidité dans la flamme, et peut être facilité du fait que les énergies d'excitation de ces bandes sont plus faibles que pour les états électroniques de l'argon et de l'hélium.

Pour la température du dard, nous avons eu principalement recours à la structure rotationnelle de $\mathrm{N}_{2}^{+}\left(\mathrm{B}, v^{\prime}=0 \rightarrow \mathrm{X}, v^{\prime \prime}=0\right)$, premier système négatif. La température rotationnelle de 
cette structure s'obtient à partir d'un graphique de Boltzmann des intensités des raies de la branche $R$. Ces raies rotationnelles sont bien résolues, étant observées avec une résolution de $0,01 \mathrm{~nm}$ pour les nombres quantiques supérieurs à 10 de cette branche. Nous avons vérifié que les températures ainsi mesurées étaient voisines de celles déterminées à partir des transitions de $\mathrm{CN}\left(\mathrm{B}, v^{\prime}=0 \rightarrow \mathrm{X}, v^{\prime \prime}=0\right)$ et $\mathrm{N}_{2}\left(\mathrm{C}, v^{\prime}=0 \rightarrow \mathrm{B}, v^{\prime \prime}=2\right)$, dans la limite de l'incertitude $\Delta T_{\mathrm{R}}= \pm 500 \mathrm{~K}$ de la mesure à partir de $\mathrm{N}_{2}^{+}$. Dans la suite, l'incertitude nıoyenne sur $T_{\mathrm{R}}$ est estimée à $500 \mathrm{~K}$ dans le domaine $2500-5500 \mathrm{~K}$ et la position axiale est connue à $\pm 0,5 \mathrm{~mm}$; les mesures de $T_{R}$ sont des valeurs moyennes d'après l'intensité totale émise selon un diamètre du dard.

Nous avons par ailleurs retenu la structure rotationnelle de $\mathrm{OH}\left(\mathrm{A}, v^{\prime}=0 \rightarrow \mathrm{X}, v^{\prime \prime}=0\right)$ uniquement pour caractériser la température du panache car cette transition n'est plus un thermomètre sensible au-delà de $1800 \mathrm{~K}$ (voir plus loin, paragraphe 3.1.2). La résolution spectrale dont nous disposions à l'époque de cette mesure $(0,045 \mathrm{~nm}$ pour une ouverture de fente de $100 \mu \mathrm{m})$ ne nous permettait pas de distinguer suffisamment les éléments des bandes rotationnelles de $\mathrm{OH}$ pour en tirer directement $T_{\mathrm{R}}$ par un diagramme de Boltzmann. Nous avons donc procédé à l'inverse, en reconstruisant par calcul le spectre de $\mathrm{OH}$ en fonction de $T_{\mathrm{R}}$ avec un niveau de résolution correspondant à celui de nos mesures. La comparaison de ce jeu de spectres théoriques avec le spectre expérimental permet de trouver assez précisément la valeur de $T_{\mathrm{R}}$ observée. Le calcul des intensités théoriques des différentes branches $(P, Q, R$ et $O$, branches principales et satellites) de la zone spoctrale étudiée a été réalisé grâce aux données de Dieke et Crosswhite [11]. Le programme informatique effectuant ces calculs suivis de la convolution des spectres avec la fonction d'appareil nous a été aimablement transmis par N. Sadeghi [12].

2.3.2. Densité électronique. - Pour caractériser l'influence des conditions de décharge et du niveau de puissance micro-onde sur la densité électronique de la flamme, nous avons eu recours à la mesure de l'élargissement Stark de la raie Balmer $\mathrm{H}_{\beta}(486,1 \mathrm{~nm})$ de l'atome d'hydrogène, élargissement produit par les micro-champs des particules chargées. Cette méthode de diagnostic est bien connue [13] ; la relation de la densité électronique $n_{\mathrm{e}}$ à la largeur $\Delta \lambda_{\mathrm{s}}$ à mi-hauteur de la raie $\mathrm{H}_{\beta}$ peut s'exprimer de façon empirique avec des polynômes [14]. Au préalabl , on aura retiré de la largeur à mi-hauteur mesurée, les contributions de l'élargissement Doppler et de la fonction d'appareil.

Nous avons évalué la fonction d'appareil à partir de la largeur de la raie $\mathrm{Ne} I 632,8 \mathrm{~nm} \mathrm{du}$ laser He-Ne à la sortie du spectromètre. Pour des fentes d'entrée et de sortie de $70 \mu \mathrm{m}$ de largeur assujetties à un spectromètre à réseau de $1 \mathrm{~m}$ de distance focale, ceci nous a conduit à une largeur d'appareil d'environ 0,03 nm. Quant à la contribution de l'élargissement Doppler, nous l'avons estimée à l'aide des températures rotationnelles mesurées dans les mêmes conditions expérimentales : pour une température du gaz de $4000 \mathrm{~K}$, cette largeur est de 0,02 $\mathrm{nm}$ pour la raie $\mathrm{H}_{\beta}$. Dans le cas d'une densité électronique de $10^{15} \mathrm{~cm}^{-3}$, la convolution de ces élargissements avec la largeur Stark correspondante donne une largeur totale à mi-hauteur d'environ $0,2 \mathrm{~nm}$; les contributions d'appareil et d'effet Doppler sont alors négligeables par rapport à l'élargissement Stark et la précision du diagnostic est grande. Par contre, pour des densités plus faibles $\left(5 \times 10^{13} \mathrm{~cm}^{-3}\right)$ et des températures $T_{\mathrm{R}}$ élevées $(5000 \mathrm{~K})$, les contributions de l'élargissement Stark $(\approx 0,03 \mathrm{~nm})$, de la fonction d'appareil $(\approx 0,03 \mathrm{~nm})$ et de l'effet Doppler $(\approx 0,025 \mathrm{~nm})$ à la convolution d'ensemble sont d'importance comparable ; dans ce cas, la valeur de la densité électronique que l'on déduit dépend de façon importante de la précision des mesures de $T_{R}$ et de la largeur d'appareil ainsi que de la forme spectrale donnée à chaque contribution dans le processus de déconvolution. Les résultats obtenus dans cette gamme de densité, i.e. à la limite de sensibilité du diagnostic, doivent être considérés à titre indicatif seulement. 
a) Torche-tige

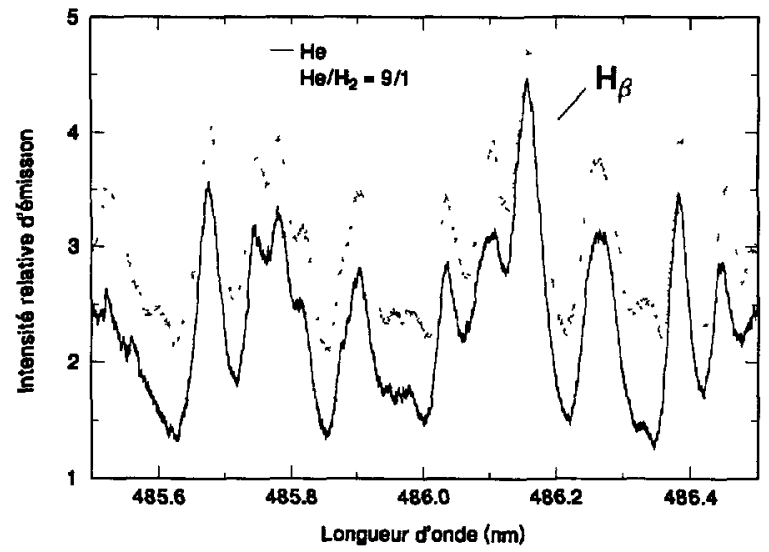

b) TIA

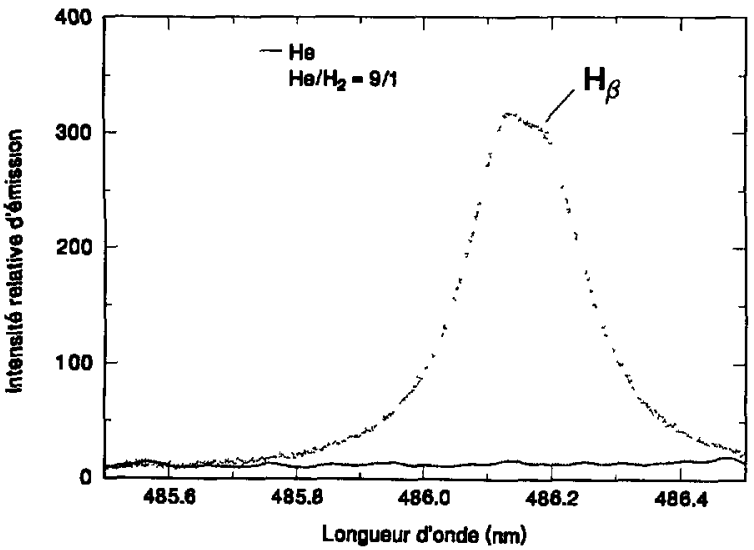

Fig. 4. - Intensité relative d'émission au voisinage de la raie $\mathrm{H}_{\beta}$ dans le dard $(z=1 \mathrm{~mm})$ avec comme gaz plasmagène de l'hélium soit pur (trait plein), soit en mélange avec $10 \%$ de $\mathrm{H}_{2}$ (pointillé) : a) pour la torche-tige ; b) pour la TIA.

[Relative emission intensity in the vicinity of the $\mathrm{H}_{\beta}$ line in the tongue $(z=1 \mathrm{~mm})$ with either pure helium (full line) or a mixture of helium with $10 \% \mathrm{H}_{2}$ (dotted line) as the carrier gas: a) for the rod-torch; b) for the TIA.]

Dans le cas de la torche-tige, l'hydrogène provenant de l'humidité de l'air présent dans la flamme suffit pour produire la raie $\mathrm{H}_{\beta}$, quoiqu'avec une intensité faible. L'ajout de $\mathrm{H}_{2}$ au gaz plasmagène n'affecte guère l'intensité de $\mathrm{H}_{\beta}$, comme on peut le voir sur la figure 4a pour un mélange de $10 \%$ vol. $\mathrm{H}_{2}-90 \%$ vol. He. On peut d'ailleurs noter qu'avec ce type de torche, la raie $\mathrm{H}_{\beta}$ avec et sans ajout de $\mathrm{H}_{2}$ au gaz plasmagène se distingue à peine des raies environnantes provenant probablement de $\mathrm{N}_{2}$ ou de $\mathrm{OH}\left({ }^{2}\right)$. On peut ainsi penser que le mélange de gaz arrivant par la douche a du mal à se rendre à la surface de la tige. A l'inverse, dans le cas de la

$\left({ }^{2}\right)$ Nous avons vérifié, au moyen d'un ajout encore plus important de $\mathrm{H}_{2}$ dans le gaz plasmagène, que la raie identifiée sur la figure $4 \mathrm{a}$ correspondait bien à $\mathrm{H}_{\beta}$. 


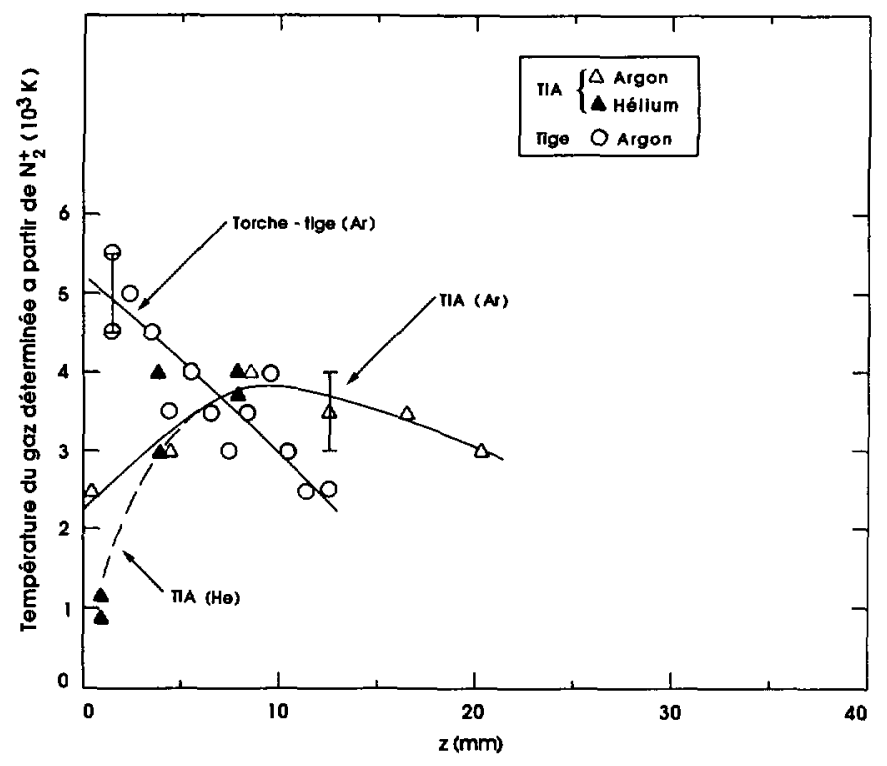

Fig. 5. - Température du gaz en fonction de la position axiale, au long du dard de la torche-tıge (argon dans la douche) et de la TIA (argon, et hélium (tirets)) à $5 \mathrm{l} / \mathrm{min}$ avec $700 \mathrm{~W}$. Nous supposons que cette température est donnée par la température rotationnelle dans la branche $\mathrm{R}$ de la transition $\mathrm{N}_{2}^{+}\left(\mathrm{B}, v^{\prime}=0 \rightarrow \mathrm{X}, v^{\prime \prime}=0\right)$.

[Gas temperature as a function of position along the tongue of the rod-torch (with argon through the shower) and of the TIA (argon, and helium (dashed line)) at $5 \mathrm{l} / \mathrm{min}$ with $700 \mathrm{~W}$. We assume this temperature to be given by the rotational temperature in the $\mathrm{R}$ branch of the $\mathrm{N}_{2}^{+}\left(\mathrm{B}, v^{\prime}=0 \rightarrow\right.$ $\mathrm{X}, v^{\prime \prime}=0$ ) transition.]

TIA où l'air environnant se mêle peu à la flamme, la mesure de l'effet Stark sans ajout de $\mathrm{H}_{2}$ est impossible à cause de la trop faible intensité de $\mathrm{H}_{\beta}$; en revanche. l'addition de seulement $10 \%$ vol. de $\mathrm{H}_{2}$ au gaz plasmagène augmente radicalement l'émission de $\mathrm{H}_{\beta}$, comme le montre la figure $4 \mathrm{~b}$. Cette raie domine alors tout le spectre d'émission dans cette fenêtre et permet une mesure précise de la densité électronique.

\section{Résultats expérimentaux}

\subsection{TEMPÉRATURE DU GAZ}

3.1.1. Température dans le dard. - Comme nous l'avons vu plus haut, le spectre des bandes rotationnelles de $\mathrm{N}_{2}^{+}(\mathrm{B})$ nous conduit à une température rotationnelle $T_{\mathrm{R}}$ que nous assimilons à la température du gaz. Nous avons ainsi déterminé la distribution axiale de la température du gaz (à partir de l'extrémité de la tige ou de la buse, selon le cas). La figure 5 reproduit les résultats obtenus pour les deux configurations de torche, à une puissance de $700 \mathrm{~W}$ et pour un débit d'argon de $5 \mathrm{l} / \mathrm{min}$. Dans le cas de la torche-tige, nous constatons que cette température est la plus élevée au voisinage immédiat de l'applicateur $(z=1 \mathrm{~mm})$ et qu'elle décroît quasilinéairement avec $z$. Examinons plus particulièrement le long de cette décharge le segment de flamme dont la température est supérieure à $2500 \mathrm{~K}$. Ce segment "chaud", comme nous le verrons, se confond avec le dard, qui se définit comme la partie de la flamme de plus grande intensité lumineuse, et de contour bien net : dans les conditions de la figure 5 , ce segment 


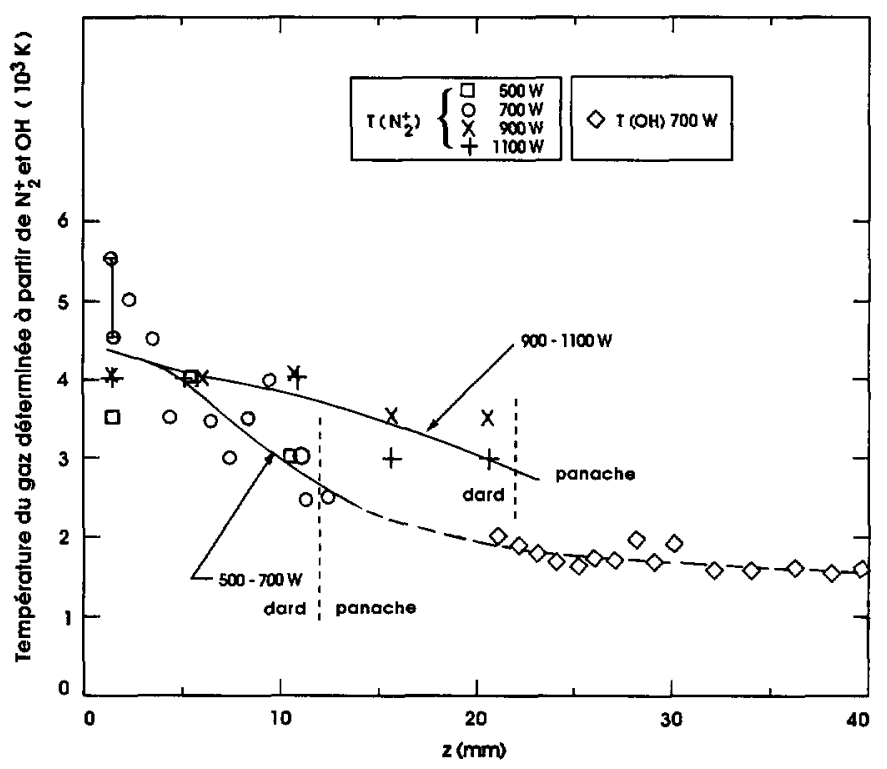

Fig. 6. - Température du gaz le long de la flamme de la torche-tige, pour diverses valeurs de la puissance micro-onde avec un débit d'argon de $5 \mathrm{l} / \mathrm{min}$ par la douche. Nous supposons que la température du gaz dans le dard et dans le panache est donnée par la température rotationnelle respectivement de la transition $\mathrm{N}_{2}^{+}\left(\mathrm{B}, v^{\prime}=0 \rightarrow \mathrm{X}, v^{\prime \prime}=0\right)$ et de la transition $\mathrm{OH}\left(\mathrm{A}, v^{\prime}=0-\mathrm{X}, v^{\prime \prime}=0\right)$.

[Gas temperature along the flame of the rod-torch, at various microwave powers with argon flowing at a rate of $5 \mathrm{l} / \mathrm{min}$ through the shower. We assume that the gas temperature in the tongue and in the plume is given by the rotational temperature of the $\mathrm{N}_{2}^{+}\left(\mathrm{B}, v^{\prime}=0 \rightarrow \mathrm{X}, v^{\prime \prime}=0\right)$ transition and of the $\mathrm{OH}\left(\mathrm{A}, v^{\prime}=0 \rightarrow \mathrm{X}, v^{\prime \prime}=0\right)$ transition respectively.]

fait environ $13 \mathrm{~mm}$ de longueur. Par contre dans la TIA, comme le montre la figure $5, T_{\mathrm{R}}$ est beaucoup plus faible au début du dard, augmentant avec $z$ pour passer par un maximum et décroître ensuite lentement. La longueur du segment "chaud" de la TIA est une fois et demie à deux fois plus grande que celle de la torche-tige, mais le maximum de température atteint est plus faible. Avec la torche-tige, l'ajout d'hydrogène (jusqu'à $20 \%$ vol.) à l'argon modifie peu les valeurs de la température, tout au moins dans la limite de la précision de nos mesures; nous reviendrons sur l'interprétation à donner à ce peu de sensibilité au gaz plasmagène pour la torche-tige.

Des essais réalisés en alimentant la TIA avec de l'hélium ont montré que la température rotationnelle de $\mathrm{N}_{2}^{+}(\mathrm{B})$ conduisait à un même profil axial que dans l'argon mais atteignait des valeurs plus basses à la sortie immédiate de la buse (voir la Fig. 5). Dans le cas de la torche-tige, la pulvérisation du métal (W) s'est avérée beaucoup plus forte avec l'hélium qu'avec l'argon comme gaz plasmagène, produisant une émission de raies atomiques de $W$ qui recouvrait celle des bandes de $\mathrm{N}_{2}^{+}(\mathrm{B})$, empêchant ainsi la mesure de $T_{\mathrm{R}}\left(\mathrm{N}_{2}^{+}\right)$.

Une augmentation de puissance permet d'accroître la longueur du segment "chaud" ; ainsi, à titre d'exemple, dans le cas de la torche-tige, cette longueur passe d'environ $13 \mathrm{~mm}$ pour 500$700 \mathrm{~W}$ à $25 \mathrm{~mm}$ pour 900-1 $100 \mathrm{~W}$ avec de l'argon comme gaz plasmagène, comme le montre la figure 6.

Quintero et al. [15] ont aussi étudié $T_{\mathrm{R}}$ dans le dard de la TIA (de $z=0$ à $z=10 \mathrm{~mm}$ ), avec l'hélium comme gaz plasmagène ; ils ont eu recours à la branche $\mathrm{P}$ de $\mathrm{N}_{2}^{+}$. Leur profil axial de 
$T_{\mathrm{R}}$ apparaît semblable au nôtre d'après ce que nous pouvons en juger (leurs mesures s'arrêtent à $z=10 \mathrm{~mm}$ alors que les nôtres s'étendent jusqu'à $20 \mathrm{~mm}$ ) : $T_{\mathrm{R}}$ croît à partir de la buse pour atteindre un maximum suivi d'une décroissance ; ce maximum se déplace vers de plus grandes valeurs de $z$ lorsqu'on augmente la puissance fournie à la torche. Cependant, leur valeur en $z \approx 0$ est plus forte que la nôtre, probablement du fait d'un débit de gaz plus faible $(3 \mathrm{l} / \mathrm{min}$ au lieu de $5 \mathrm{l} / \mathrm{min}$ ) : ainsi, ils obtiennent en ce point de 2000 à $2400 \mathrm{~K}$ pour une puissance de 300 à $900 \mathrm{~W}$ alors que nous y avons relevé $1000 \mathrm{~K}$ pour $700 \mathrm{~W}$ avec le même gaz.

3.1.2. Température dans le panache. - Nous supposons qu'elle est donnée par la température $T_{\mathrm{R}}$ du spectre rotationnel de la transition $\mathrm{OH}(\mathrm{A} \rightarrow \mathrm{X})$. Comme nous l'avons dit plus haut (paragraphe 2.3.1), c'est par la comparaison du spectre expérimental avec les spectres synthétiques correspondants que nous déduisons $T_{\mathbf{R}}$. A titre d'exemple de cette méthode, comparons le spectre expérimental de la figure $7 \mathrm{a}$ avec les spectres reconstruits des figures $7 \mathrm{~b}$ et $7 \mathrm{c}:$ on voit que le spectre expérimental est proche du spectre synthétique à $T_{\mathrm{R}}=1600 \mathrm{~K}$. Les valeurs de $T_{\mathrm{R}}$ ainsi obtenues sont reportées sur la figure 6 . Nous constatons que $T_{\mathrm{R}}$ diminue lentement en fonction de $z$ dans le panache de la torche-tige (argon $5 \mathrm{l} / \mathrm{min}, 700 \mathrm{~W}$ ), passant de $2000 \mathrm{~K}$ pour $z=20 \mathrm{~mm}$ à $1500 \mathrm{~K}$ pour $z=40 \mathrm{~mm}$.

Si nous comparons maintenant les spectres synthétiques des figures $7 \mathrm{~b}$ et $\mathrm{c}$ avec ceux des figures $8 \mathrm{a}$, b et c, nous constatons qu'il y a peu d'évolution du spectre entre 2200 et $4200 \mathrm{~K}$ : en effet, pour $T_{R} \leq 1600 \mathrm{~K}$, l'intensité du doublet situé entre 306 et $307 \mathrm{~nm}$ est nettement plus faible que l'intensité du reste de la structure de 308 à $311 \mathrm{~nm}$ alors que ce n'est plus le cas dans l'intervalle de $T_{\mathrm{R}}$ entre 2200 et $4200 \mathrm{~K}$, les deux structures demeurant d'intensités à peu près égales, avec une très légère croissance de l'intensité du doublet relativement au reste de la structure lorsque $T_{\mathrm{R}}$ augmente. L'examen d'un ensemble plus complet de spectres synthétiques nous amène à conclure plus précisément que la structure rotationnelle de $\mathrm{OH}(\mathrm{A} \rightarrow \mathrm{X})$ n'est pas un thermomètre sensible pour $T_{R} \geq 1800 \mathrm{~K}$. La variation relative des intensités des raies marquées d'une croix dans les figures 7 et 8 est trop faible pour permettre d'attribuer, avec précision, une valeur de $T_{\mathrm{R}}$ pour $T_{\mathrm{R}} \geq 1800 \mathrm{~K}$.

3.2. Densité Électronique. - La méthode décrite au paragraphe 2.3.2 nous a permis d'établir la distribution axiale de la densité électronique, $n_{\mathrm{e}}(z)$, de la flamme des deux torches. La figure 9 montre cette distribution pour un mélange plasmagène $\mathrm{Ar} / \mathrm{H}_{2}=4 / 1$, un débit total de $5 \mathrm{l} / \mathrm{min}$ et ule puissance injectée de $700 \mathrm{~W}$. On remarquera que la densité du plasma tout près de l'applicateur ( $z \approx 1 \mathrm{~mm}$ ) est beaucoup plus élevée avec la TIA qu'avec la torche-tige : $2,2 \times 10^{15} \mathrm{~cm}^{-3}$ contre $6,5 \times 10^{13} \mathrm{~cm}^{-3}$; cependant, la densité dans le cas de la TIA décroît très rapidement en s'éloignant, au contraire de ce qui se passe avec la torche-tige. Le fait que le plasma de la torche-tige soit plus long que celui de la TIA correspond à une répartition spatiale différente des espèces chargées car on peut supposer qu'en première approximation la puissance requise pour créer une paire électron-ion est la même dans les deux types de décharge $\left({ }^{3}\right)$.

La figure 9 permet aussi de voir l'influence sur $n_{\mathrm{e}}$ de l'ajout d'hydrogène au gaz plasmagène (argon) dans le cas de la torche-tige $\left({ }^{4}\right)$ : l'augmentation de $n_{\mathrm{e}}$ est faible, cette différence se distinguant à peine de l'incertitude sur la mesure de ces faibles densités (paragraphe 2.3.2). Cet effet négligeable est conforme à ce qui a été rapporté plus haut (Fig. 4a), à savoir que l'addition de $\mathrm{H}_{2}$ a peu d'influence sur l'intensité d'émission de la raie $\mathrm{H}_{\beta}$ dans le cas de la torche-tige.

$\left({ }^{3}\right)$ Cette affirmation doit cependant être nuancée pour tenir compte du rôle important de $\mathrm{N}_{2}$ dans le plasma de la torche-tige (même si le gaz plasmagène est le même dans les deux configurations) et aussi de mécanismes différents de recombinaison des particules chargées.

$\left({ }^{4}\right)$ La figure 4 b nous a enseigné que l'observation de la raie $H_{\beta}$ dans la TIA est tout à fait impossible sans cette addition de $\mathrm{H}_{2}$. 


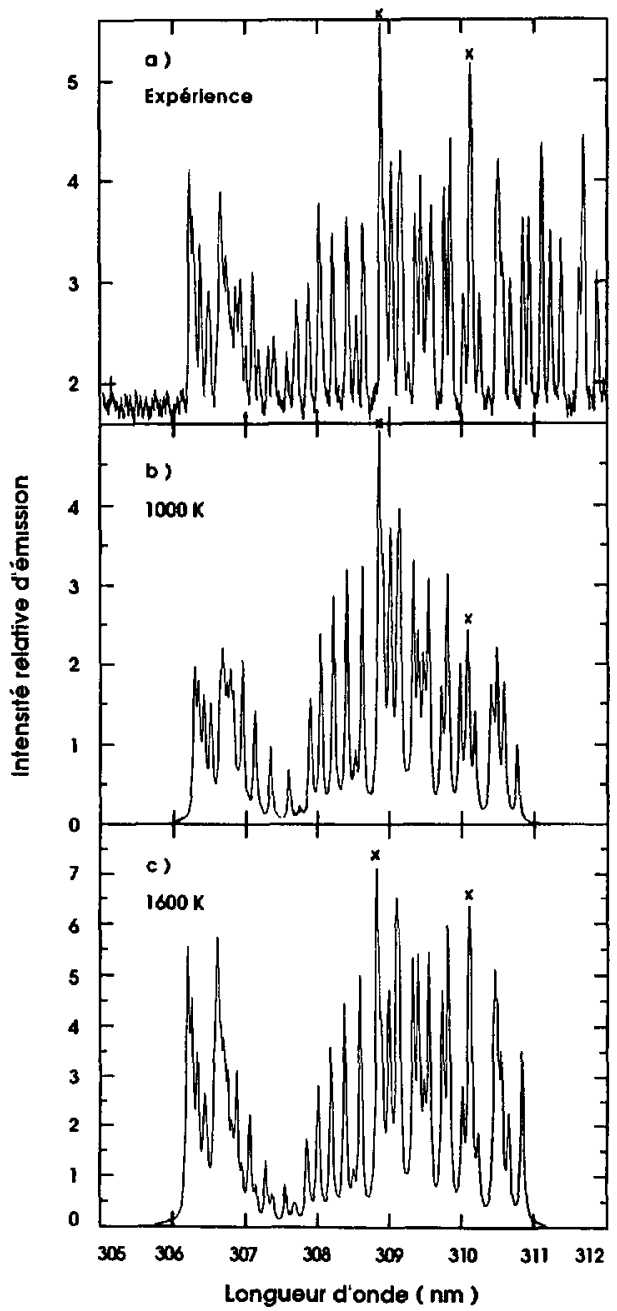

Fig. 7. - Exemple d'utilisation des spectres synthétiques pour déterminer la valeur expérimentale de la température rotationnelle $T_{\mathrm{R}}$ de la transition $\mathrm{OH}\left(\mathrm{A}, v^{\prime}=0 \rightarrow \mathrm{X}, v^{\prime \prime}=0\right):$ a) spectre expérimental obtenu avec la torche-tige $\left(25 \% \mathrm{H}_{2}-75 \% \mathrm{Ar}, 5 \mathrm{l} / \mathrm{min}, 700 \mathrm{~W}\right.$ à $\left.z=34 \mathrm{~mm}\right)$; b) et c) : spectres synthétiques de même résolution spectrale calculés pour $T_{\mathrm{R}}=1000$ et $1600 \mathrm{~K}$ respectivement. La détermination de $T_{R}$ se fait en comparant l'intensité du doublet situé entre 306 et $307 \mathrm{~nm}$ par rapport à l'intensité du reste de la structure rotationnelle ; les raies surmontées d'une croix sur la figure servent de points de repère dans cette comparaison. Nous en déduisons que $T_{\mathrm{R}}$ (expérience) $\approx 1600 \mathrm{~K}$ dans le cas présent.

[An example of how to use synthetic spectra for determining the rotational temperature of the $\mathrm{OH}$ $\left(\mathrm{A}, v^{\prime}=0 \rightarrow \mathrm{X}, v^{\prime \prime}=0\right)$ transition: a) experimental spectrum obtained with the rod-torch $\left(25 \% \mathrm{H}_{2}\right.$ $75 \% \mathrm{Ar}, 5 \mathrm{l} / \mathrm{min}, 700 \mathrm{~W}$ at $z=34 \mathrm{~mm}$ ); b) and c): synthetic spectra with the same spectral resolution, as calculated for $T_{\mathrm{R}}=1000$ and $1600 \mathrm{~K}$ respectively. One compares the intensity of the doublet lying between 306 and $307 \mathrm{~nm}$ relatively to the rest of the rotational structure; the lines surmounted by a cross serve as points of reference for this comparison. We conclude that the observed $T_{\mathrm{R}}$ is close to $1600 \mathrm{~K}$ in the present case.] 


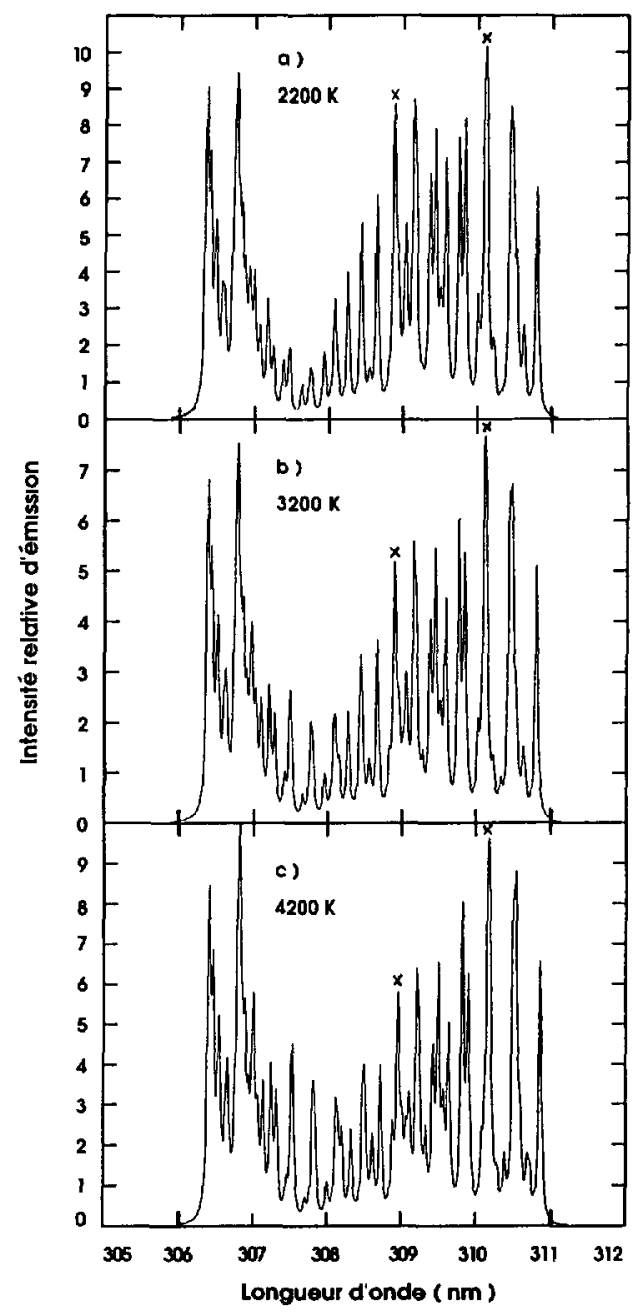

Fig. 8. - Évolution des spectres synthétiques en fonction de la température rotationnelle $T_{\mathrm{R}}$ de la transition $\mathrm{OH}\left(\mathrm{A}, v^{\prime}=0 \rightarrow \mathrm{X}, v^{\prime \prime}=0\right)$ : a) $2200 \mathrm{~K}$; b) $3200 \mathrm{~K}$; c) $4200 \mathrm{~K}$. En comparant avec les spectres synthétiques de même résolution pour $T_{R}=1000$ et $1600 \mathrm{~K}$ des figure $7 \mathrm{~b}$ et $c$ et en suivant la démarche qui y est décrite, nous constatons que la structure rotationnelle évolue de moins en moins avec $T_{\mathrm{R}}$ croissant.

[Evolution of synthetic spectra as a function of the rotational temperature $T_{\mathrm{R}}$ of the $\mathrm{OH}\left(\mathrm{A}, v^{\prime}=0 \rightarrow\right.$ $\mathrm{X}, v^{\prime \prime}=0$ ) transition: a) $2200 \mathrm{~K}$; b) $3200 \mathrm{~K}$; c) $4200 \mathrm{~K}$. By comparing with the corresponding synthetic spectra for $T_{\mathrm{R}}=1000$ and $1600 \mathrm{~K}$ in Figures $7 \mathrm{~b}$ and $\mathrm{c}$, and by following the procedure described therein, we notice that the changes in the rotational structure are less and less significant as $T_{R}$ increases.]

De ces observations, nous concluons que la flamme de cette torche a son comportement principalement déterminé par l'air environnant, le gaz plasmagène intervenant peu ; l'hydrogène atomique présent dans la flamme proviendrait donc plutôt de l'humidité de l'air.

En comparant les distributions $n_{e}(z)$ des figures 9 et 10 pour le cas de la TIA, on constate que malgré la nature différente des gaz plasmagènes et leurs débits différents $\left(\mathrm{Ar} / \mathrm{H}_{2}=4 / 1\right.$ 


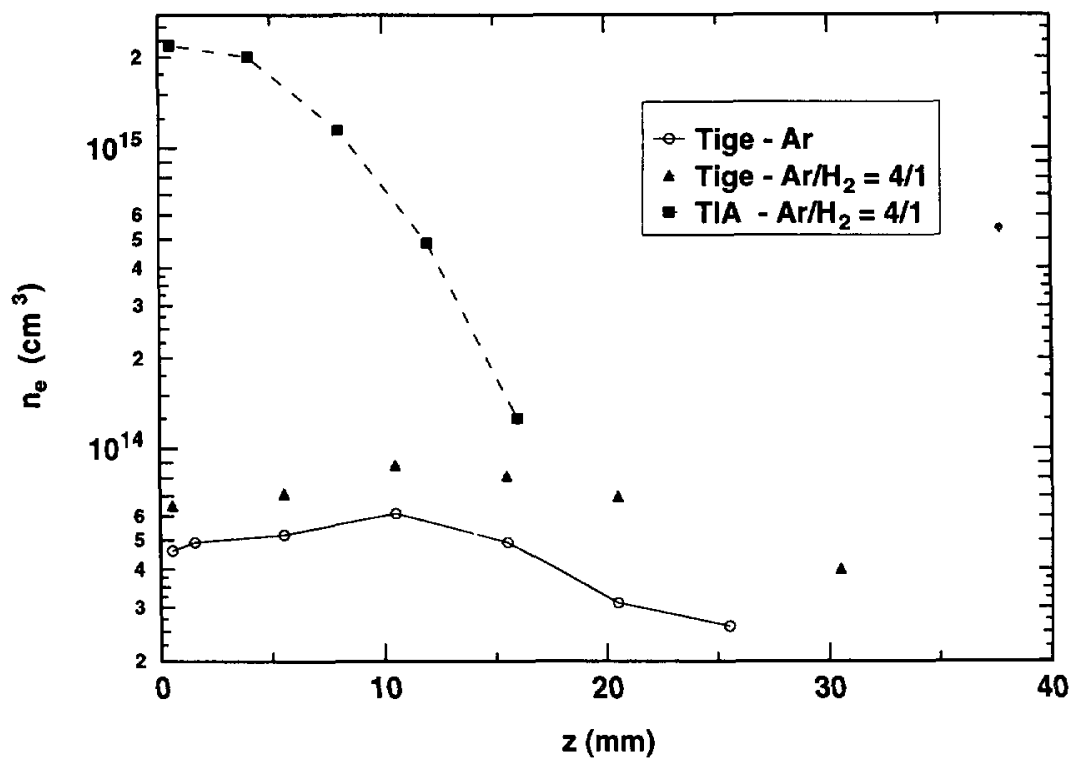

Fig. 9. - Densité électronique observée en fonction de la position axiale dans la flamme des deux

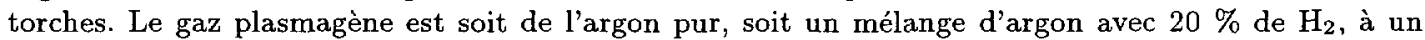
débit total de $5 \mathrm{l} / \mathrm{min}$; la puissance micro-onde est de $700 \mathrm{~W}$.

[Electron density as a function of axial position in the flame of both torches. The carrier gas is either pure argon, or argon mixed with $20 \% \mathrm{H}_{2}$, at a total flow rate of $5 \mathrm{l} / \mathrm{min}$; the microwave power is set at $700 \mathrm{~W}$.]

et $5 \mathrm{l} / \mathrm{min}$ dans un cas, et $\mathrm{He} / \mathrm{H}_{2}=9 / 1$ avec $10 \mathrm{l} / \mathrm{min}$ dans l'autre), la densité électronique près de l'extrémité de la buse ainsi que la longueur du plasma $\left(\approx 2 \times 10^{15} \mathrm{~cm}^{-3}\right.$ et $\approx 20 \mathrm{~mm}$ respectivement) demeurent très semblables pour une puissance donnée $(700 \mathrm{~W})$.

\section{Discussion et conclusion}

4.1. DARD ET PANACHE. - Dans notre description de la structure axiale de la flamme, nous avons utilisé tantôt le terme dard ou panache, tantôt la notion de segment "chaud", et enfin le terme de plasma qui s'entend d'un milieu contenant des particules chargées. Nous allons maintenant préciser les relations entre ces différentes désignations.

La figure 10 montre le profil axial de l'intensité spectrale totale provenant de la raie $\mathrm{H}_{\beta}$. Cette émission décroît très rapidement à l'approche de la position axiale où la densité électronique tend vers zéro, dans le cas présent vers $z \approx 20 \mathrm{~mm}$. Cette même position axiale correspond aussi d'assez près à la fin du dard, qui est la partie de forte luminosité de la flamme. Enfin, nous reportant à la figure 5 , nous constatons que la longueur du segment "chaud" $\left(T_{\mathrm{R}}>2500 \mathrm{~K}\right)$ est voisine de la longueur du dard. Nous en concluons : i) que l'émission $\mathrm{H}_{\beta}$, tout au moins dans le cas de la TIA, est représentative de l'émission lumineuse de la majorité des espèces émettrices présentes dans le dard ; ii) que le dard délimite bien l'extension spatiale du plasma ; si. le panache de la TIA comporte des particules chargées, leur densité est faible, inférieure à notre limite de mesure par $\mathrm{H}_{\beta}\left(3 \times 10^{13} \mathrm{~cm}^{-3}\right)$; et iii) que le segment "chaud" tel que défini correspond bien au dard. 


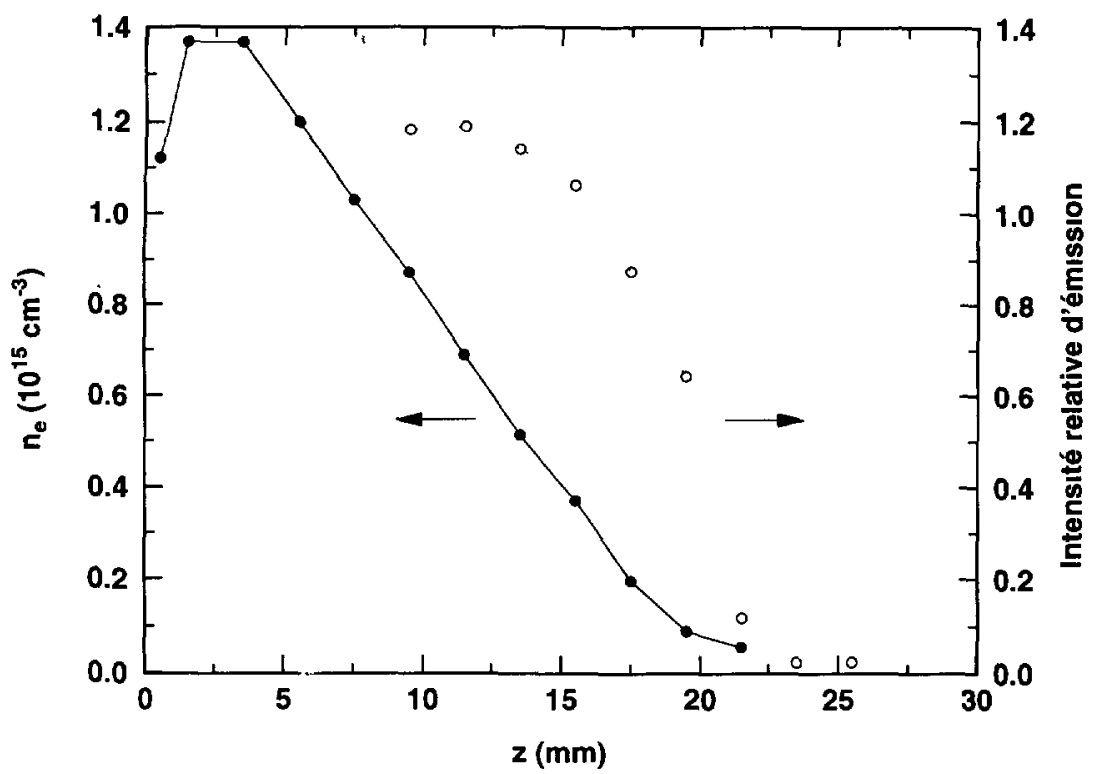

Fig. 10. - Densité électronique observée en fonction de la position axiale dans le dard de la TIA (échelle de gauche) ; et intensité spectrale totale de la raie $\mathrm{H}_{\beta}$ en fonction de la position axiale (échelle de droite). Le gaz plasmagène est un mélange $\mathrm{He} / \mathrm{H}_{2}=9 / 1$ à un débit total de $10 \mathrm{l} / \mathrm{min}$, la puissance micro-onde étant de $700 \mathrm{~W}$.

[Electron density as a function of axial position in the tongue of the TIA (lefthand scale); total spectral intensity of the $\mathrm{H}_{\beta}$ line as a function of the axial position (righthand scale). The carrier gas is a $\mathrm{He} / \mathrm{H}_{2}=9 / 1$ mixture at a total flow rate of $10 \mathrm{l} / \mathrm{min}$, the microwave power being set at $700 \mathrm{~W}$.]

4.2. Mécanismes D'ionisation et de Chauffage du Gaz. - Dans le cas de la Tia, l'ionisation est la plus grande à l'extrémité même de la buse où le flux micro-onde est maximum, et elle résulte complètement des électrons accélérés dans le champ électrique micro-onde. De ce fait, le flux micro-onde décroît rapidement axialement, d'où la décroissance observée de $n_{\mathrm{e}}$. Cette ionisation a lieu dans le gaz plasmagène qui est en écoulement rapide de sorte que le chauffage du gaz, résultant de transferts d'excitation et de la recombinaison en volume, atteint son maximum un peu plus loin dans la décharge.

Les mécanismes sont différents pour la torche-tige. Le flux micro-onde est aussi maximum à l'extrémité de l'applicateur mais il décroît lentement car l'ionisation du gaz est partiellement assurée par l'émission thermo-ionique de l'extrémité de la tige qui est portée à haute température, voire au point de fusion. De plus, cette ionisation a lieu principalement dans le gaz qui stagne devant la section droite de l'extrémité de la tige : pour le gaz plasmagène venant de la douche, la tige définit un écoulement qui présente en effet un point de stagnation à la surface de l'extrémité de la tige. L'écoulement rapide du gaz plasmagène pourrait même créer une dépression à l'extrémité de la tige, cette réduction de pression favorisant l'ionisation en ce point.

4.3. ECART À L'ETL. - Un autre point différenciant les deux torches est leur degré d'écart à l'équilibre thermodynamique local (ETL) dans le dard. Considérons d'abord le cas de la TIA : par exemple, avec l'argon comme gaz plasmagène, la densité du plasma peut atteindre expérimentalement $2 \times 10^{15} \mathrm{~cm}^{-3}$, ce qui correspondrait en ETL à une température du plasma 
de $8000 \mathrm{~K}[16]$; expérimentalement, nous trouvons une température $T_{\mathrm{R}}$ de gaz inférieure à $4000 \mathrm{~K}$, donc nous sommes loin de l'ETL. Avec l'hélium comme gaz plasmagène, la densité expérimentale est encore voisine de $2 \times 10^{15} \mathrm{~cm}^{-3}$, ce qui nécessiterait cette fois une température de plasma de $13300 \mathrm{~K}$ pour l'ETL ; expérimentalement, $T_{\mathbf{R}} \leq 4000 \mathrm{~K}$ : le plasma d'hélium de la TIA est encore plus loin de l'ETL que celui d'argon. Par contre dans le cas de la torchetige, la densité du plasma dans le dard est plus faible, au plus $9 \times 10^{13} \mathrm{~cm}^{-3}$ Si nous faisons l'hypothèse que la flamme est contrôlée soit par l'argon que nous introduisons par la douche soit au contraire par l'azote de l'air, il faudrait respectivement une température de plasma de 6500 et de $6600 \mathrm{~K}$ pour qu'il y ait ETL ; expérimentalement, nous atteignons au maximum un peu plus de $5000 \mathrm{~K}$ pour $T_{\mathrm{R}}$ : l'écart à l'ETL de la torche-tige est nettement plus faible quand on le compare à celui de la TIA.

4.4. MÉRITES RESPECTIFS DES DEUX TORChES. - Le fait que la flamme de la torche-tige soit plus près de l'ETL que celle de la TIA peut constituer un avantage dans la mesure où l'on recherche un plasma "thermique", soit pour des études fondamentales, soit pour des applications. De plus, sachant que le chauffage de l'extrémité de la tige engendre une émission de raies, on pourrait envisager l'usage de cette torche pour l'analyse chimique de traces d'impureté dans des matériaux conducteurs.

La TIA présente elle aussi un certain nombre d'avantages qui lui sont propres. Elle permet d'injecter si nécessaire les substances à transformer directement dans l'axe du plasma, ce qui peut s'avérer un avantage par rapport à une injection coaxiale (comme c'est le cas pour l'applicateur à tige) ou à une injection transversale à l'axe du plasma (par exemple, pour les arcs en courant continu) où l'interaction du produit avec le plasma est a priori hydrodynamiquement moins efficace. Enfin, l'injection axiale conduit à de plus grandes vitesses de translation axiale, ce qui est utile quand on veut projeter rapidement un produit de la phase gazeuse sur une surface. En particulier, la TIA est bien adaptée à des traitements localisés de surfaces, à grands rendements, par l'ajout au gaz plasmagène de gaz réactifs comme $\mathrm{N}_{2}, \mathrm{~N}_{2}-\mathrm{H}_{2}, \mathrm{~N}_{2}-\mathrm{CH}_{4}$ pour des opérations de nitruration d'objets métalliques de formes complexes (avec trous, fentes, etc.). Des dépôts de couches de carbone dur, adamantin ou diamant, à grande vitesse, pourraient également être obtenus en ajoutant des mélanges $\mathrm{H}_{2}-\mathrm{CH}_{4}$. Les températures obtenues avec la TIA sont élevées sur un domaine plus long qu'avec la torche-tige (Fig. 5), d'où un temps de résidence plus grand des produits dans le segment chaud, et les densités électroniques sont également plus élevées, d'où un taux de production d'espèces actives plus élevé, de sorte que ces conditions devraient permettre à la chimie dans ces décharges d'être plus efficace.

4.5. Conclusion. - Cette étude spectroscopique nous a permis de mettre en évidence les principales caractéristiques physiques de la flamme de deux torches à plasma micro-onde. Les mesures quantitatives et résolues spatialement de la température du gaz et de la densité électronique ont été corrélées à l'observation visuelle des torches, ce qui a mené à une connaissance détaillée de la structure axiale des flammes. Ces deux configurations de torche micro-onde se sont révélées complémentaires à différents points de vue. La TIA (torche à injection axiale) produit un plasma de grande densité électronique $\left(>10^{15} \mathrm{~cm}^{-3}\right)$, tandis que la flamme de la torche-tige est beaucoup moins dense quoique plus chaude ; cette dernière s'approche donc davantage de l'ETL ; par ailleurs, l'air ambiant s'incorpore plus facilement à la flamme de la torche-tige. Cette complémentarité des deux torches est renforcée par le fait que l'on peut très facilement passer de l'une à l'autre (i.e. tour à tour, la buse et la tige) à partir d'un même système d'adaptation d'impédance, système qui de plus se révèle d'une grande efficacité énergétique. Nous avons fait valoir finalement que les deux torches se prêtaient bien chacune à différentes applications dans les domaines de la physique et de la chimie. 


\section{Remerciements}

Nous tenons à remercier pour leur précieux soutien technique Messieurs François Roy, Rémy Grenier, Robert Lemay, Robert Martel et Jean-Eudes Samuel de l'Université de Montréal, où s'est déroulée la partie expérimentale. Nous sommes également redevables à $M$. Gaston Sauvé et au professeur Zenon Zakrzewski pour leurs conseils au cours de cette expérience, et à M. Nader Sadeghi pour son concours dans le calcul des spectres synthétiques de $\mathrm{OH}$. Enfin, ce travail a pu être réalisé grâce aux missions effectuées dans le cadre des accords scientifiques France-Québec (projet 02-58-02-91).

\section{Bibliographie}

[1] Boulos M.I., RF induction plasma spraying: state-of-the-art review, J. Thermal Spray Technol. 1 (1992) 33-40.

[2] Mitsuda M., Yoshida T. et Akashi K., Development of a new microwave plasma torch and its application to diamond synthesis, Rev. Scr. Instrum. 60 (1989) 249-252.

[3] Murayama M., Kojima S. et Uchida K., Uniform deposition of diamond films using a flat flame stabilized in the stagnation-point flow, J. Appl. Phys. 69 (1991) 7924-7926.

[4] Krause T.R. et Helt J.E., "Chemical detoxification of trichloroethylene and 1,1,1-trichloroethane in a microwave discharge plasma reactor at atmospheric pressure", Emerging technologies in hazardous waste management III, D. W. Tedder, G. Pohland Eds., ACS Symp. Series 518 (1993) p. 393.

[5] Boudène C., Cluet J.-L., Keib G. et Wind G., Identification et étude de quelques propriétés des composés résultant de la décomposition du $\mathrm{SF}_{6}$ sous l'effet de l'arc électrique dans les disjoncteurs, Arc/ Drsjoncteurs/Sécurté/ $S F_{6} 45$ (1974) 45-78.

[6] Jin Q., Zhu C., Borer M. et Hieftje G.M., A microwave plasma torch assembly for atomic emission spectrometry, Spectrochım. Acta 46B (1991) 417-430.

[7] Hubert J., Sing R., Boudreau D., Tran K.C., Lauzon C. et Moisan M., "Applications of microwave discharges to elemental analysis", Microwave discharges: fundamentals and applications, C. M. Ferreira, M. Moisan Eds., NATO-ASI Series B: Physics 302 (Plenum, New York, London, 1993) pp. 509-530.

[8] Moisan M., Ferreira C.M., Hajlaoui Y., Henry D., Hubert J., Pantel R., Ricard A. et Zakrzewski Z., Properties and applications of surface wave produced plasmas, Rev. Phys. Appl. 17 (1982) 707-727.

[9] Besner A., Hubert J. et Moisan M., Fundamental properties of radiofrequency and microwave surface-wave induced plasmas, J. Anal. At. Spectrom. 3 (1988) 863-866.

[10] Moisan M., Sauvé G., Zakrzewski Z. et Hubert J., An atmospheric pressure waveguide-fed microwave plasma torch: the TIA design, Plasma Sources Sci. Technol. 3 (1994) 584-592.

[11] Dieke G.H. et Crosswhite H.M., The ultraviolet bands of OH-fundamental data, J. Quant. Spectrosc. Radiat. Transfer 2 (1962) 97-199.

[12] Sadeghi N., Communication directe (1994).

[13] Griem H.R., Plasma Spectroscopy (McGraw-Hill, New York, 1964).

[14] Coulombe S., Mise au point et application d'un détecteur à plasma micro-onde pour la chromatographie en phase gazeuse, Thèse de Ph.D., département de chimie, Université de Montréal (1982).

[15] Quintero M.C., Rodero A., Gamero A. et Sola A., "Experimental study of an atmospheric pressure helium microwave plasma torch produced by the T.I.A. structure II: rotational and excitation temperatures", Twelfth European Sectional Conference on the Atomic and Molecular Physics of Ionized Gases (ESCAMPIG 1994), Noordwijkerhout, Pays-Bas (août 1994).

[16] Boulos M.I., Fauchais P. et Pfender E., Thermal Plasmas, vol. 1 (Plenum, New York, London, 1994). 